\title{
Interactive comment on "Long-term variability of solar irradiance and its implications for photovoltaic power in West Africa” by Ina Neher et al.
}

\section{Anonymous Referee \#3}

Received and published: 12 June 2020

\section{General comments}

In this work, I can distinguish two main parts: First, an evaluation was performed for 35year time series of SARAH-2.1 global and direct surface horizontal irradiance against ground-based measurements from three stations in West Africa and the spatial and temporal variability of these data was assessed. Second part was the use of satellite data to derive empirically pv yields for West Africa region and these results were analyzed in space and time and were validated by explicitly calculated values from the aforementioned ground-based stations. As long as solar energy renewable sources is a promising and sustainable source of energy, this study provides interesting results 
concerning PV power potential in West Africa. I would consider to recommend this paper for publication, if the authors can address the following comments:

Specific comments

Lines 22-23: you state climatological conditions governing a great area, you may support it with relevant bibliography?

Interactive

comment

Lines 64-71: Apart from the study's area topography, from which I suggest to begin with, which provides the valuable information of mountain regions essential for cloud formation, you provide climatological conditions of study area especially surface albedo, mean cloud albedo, aerosol optical depth. You provide this information by figure 1 and there you explain briefly how these values are computed. Please, describe here how those values and fig 1 produced giving information in the manuscript about the data used.

Line 118: "for monthly mean temperature" maybe you mean daily mean temperature as you mention at line 124

Line 155: GTI and not GHI?

Lines 159-160: maybe: the parameter b ... . The slope $\alpha$ ?

Lines 212-213: the percentages inside parenthesis are reductions of RMSE? Are the right values because it doesn't make sense for example for Afougou compared to the values given in fig. 3

Line 285: "... being significant" please rephrase that sentence and give additional information of how you assess the statistical significance of the linear trends?

Figure 8 caption: Trends of monthly mean anomalies were calculated and provided on the plots, if they were found to be statistically significant, please provide information about how you assess the statistical significance.

Figure 10 caption: The central line of those box plots provides mean value or median? 
Please explain and if is the median perhaps you should provide on this figure the median of the explicitly calculated PV yields for the three sites.

Figure 10 caption: Instead of "temporally" temporal variations.

Technical corrections

Lines 8, 10: use parenthesis instead of $\mathrm{W} / \mathrm{m} 2 /$ decade use $(\mathrm{W} / \mathrm{m} 2) /$ decade and everywhere applicable in the manuscript

Line 31: Organization not "Organisation"

Lines 35: and for the full 35 ... instead of "and the full 35 ..."

Line 60: May not "Mai"

Line 80: measurement not "measuremten"

Line 119: and is available ... instead of "and available"

Line 181: and decreases (0.78) [without to]

Figure 2: the symbols used to describe the regression lines must be consistent through the manuscript, so please select only one symbol for the slope

Line 186: the verb is missing in order to complete your sentence, so in order to make sense please rephrase you sentence

Figure 3: At the second sentence please remove second "the".

Line 231 : . . .the temporal mean. . instead of ". . .the temporally mean. .."

Figure 4 caption: (1983 to 2017) ... instead of "(1983 and 2017)"

Figure 4: Main title of c,d: interquartile

Figure 4 caption: temporal variations instead of "temporally"

Line 253: temporal variations of IQR and not "temporally IQR" and everywhere appli- 
cable in the manuscript

Figure 5: Main title of c,d: interquartile

ACPD

Line 269: summer months. . . instead of "summer month"

line 318: ... time series of monthly mean ... instead of "... time series monthly mean ..."

Figure 9: Main title of b: interquartile

Line 386: ... is expensive. instead of "... is expansive."

Line 389: ... the urban regions in the south. instead of "... the urban regions is the south."

Interactive comment on Atmos. Chem. Phys. Discuss., https://doi.org/10.5194/acp-2020-306, 2020. 